\title{
Caracterización de la avifauna e ictiofauna asociada a los bañados de desborde fluvial de cuatro arroyos pampeanos con uso de suelo contrastante (Buenos Aires, Argentina)
}

\author{
Juan Martín Paredes del Puerto ${ }^{1}$, Ignacio Daniel García ${ }^{1}$, Roberto Francisco Jensen ${ }^{1}$, \\ Tomás Maiztegui ${ }^{1}$, Ariel Hernán Paracampo ${ }^{1}$, Javier Ricardo Garcia de Souza ${ }^{1}$, Miriam \\ Edith Maroñas ${ }^{1}$, Darío César Colautti ${ }^{1}$
}

${ }^{1}$ Instituto de Limnología “Dr. Raúl A. Ringuelet” (CONICET-UNLP), Boulevard 120 y 62, La Plata, Buenos Aires, Argentina.

E-mail: jmparedesdelpuerto@gmail.com

\begin{abstract}
RESUMEN. Las actividades humanas en los sistemas naturales promueven cambios que ponen en peligro a las comunidades acuáticas por pérdida de nichos ecológicos generando disminución de la riqueza y diversidad. El objetivo de esta investigación fue analizar los ensambles de aves y peces asociados a cuatro bañados de arroyos pampeanos con usos del suelo periurbano y rural en sus cuencas. Los resultados evidenciaron que existen importantes diferencias en la composición y estructura de dichos ensambles según la cuenca de pertenencia. Los valores más bajos de riqueza y de diversidad se registraron en los sitios periurbanos, donde además se observó la dominancia de las especies más tolerantes de ambos grupos de vertebrados. El estudio en simultáneo de estos ambientes nos permitió registrar de qué manera, en una misma región, los cambios inducidos por el hombre condicionan la configuración de los ensambles de aves y peces. En consecuencia, el análisis multi-taxa realizado no solo resulta de carácter diagnóstico, sino que también puede utilizarse como herramienta para el monitoreo, diseño e implementación de medidas de remediación.
\end{abstract}

Palabras clave: Arroyos de llanura, impacto antrópico, ensambles de aves, ensamble de peces, pérdida de biodiversidad.

\begin{abstract}
Human activities in natural systems promote changes that endanger aquatic communities due to the loss of ecological niches, generating a decrease in richness and diversity. The bird and fish assemblages of four wetlands in Pampean streams with peri-urban and rural land uses in their basins were studied. The results evidenced that there are important differences in the composition and structure of the assemblages of birds and fish according to the basin of belonging. The lowest values of richness and diversity were registered in the peri-urban places, where the dominance of the most tolerant species of both groups of vertebrates was also observed. The simultaneous study of these environments enabled records of how, in the same region, human-induced changes condition the configuration of birds and fish assemblages. Consequently, the analysis multi-taxa performed is not only a diagnostic report but can also be used as a tool for monitoring, developing and implementing remediation measures.
\end{abstract}

Keywords: Anthropic impact, biodiversity loss, bird assemblages, fish assemblages, lowland streams. 


\section{INTRODUCCIÓN}

La biodiversidad de los ambientes acuáticos continentales está disminuyendo a una tasa mayor que la de cualquier otro tipo de ambiente (Albert et al., 2020; Dudgeon et al., 2006; McGarrigle, 2014; Strayer \& Dudgeon, 2010), lo cual permite concebir a las comunidades de agua dulce como una de las más vulnerables del planeta (Albert et al., 2020; Richards et al., 1996). El deterioro de los sistemas naturales está asociado con las actividades humanas (Abell et al., 2008), que promueven cambios en la calidad del agua y ponen en peligro a las comunidades de agua dulce.

Entre las principales amenazas a la integridad ecológica de los ecosistemas, se encuentra el uso del territorio en las cuencas hidrográficas (Allan, 2004; Roth et al., 1996). Las grandes extensiones de tierras que son utilizadas por la agricultura y la ganadería provocan efectos contraproducentes sobre los ecosistemas acuáticos tales como el incremento de la turbidez, la pérdida de macrófitas, el aporte de agroquímicos, la depresión del nivel freático y la modificación de los cauces que, en conjunto con otros efectos, ocasionan la pérdida de nichos ecológicos para las especies (Blann et al., 2009; Burcher \& Benfield, 2006; Giorgi et al., 2014). Mensing et al. (1998) han reportado que la disminución de los valores de riqueza y diversidad de aves y peces se encuentra directamente relacionada con el aumento del área cultivada de las cuencas. Este efecto de empobrecimiento se manifiesta más acentuadamente en zonas urbanizadas, donde las comunidades de aves se caracterizan por exhibir una baja riqueza específica (Chace \& Walsh, 2006), al igual que los ensambles de peces que habitan los arroyos y ríos que atraviesan sectores urbanos (Gafny et al., 2000; Helms et al., 2005; Steedman, 1988).

Los procesos de ocupación y uso del territorio han avanzado sin atender de manera adecuada cuestiones vinculadas a la conservación de la biodiversidad ni a la preservación de los servicios ecosistémicos de los cuales, paradójicamente, se vale el hombre para sostener su desarrollo. Esta situación no ha pasado desapercibida y se ha ido incorporando progresivamente a la agenda política ambiental (Bateman et al., 2013). Por ello se han incrementado las investigaciones orientadas a proveer criterios para la toma de decisiones de manejo ambiental (Daily et al., 2009; De Groot et al., 2010; Goldstein et al., 2012; Maes et al., 2013). No obstante, en muchos casos es aún necesario generar líneas de base elementales para contar con conocimientos que posibiliten implementar una gestión del territorio orientada a su aprovechamiento sostenible. En ese marco, el empleo de indicadores biológicos para detectar cambios frente al desarrollo humano emerge como una pieza clave en el camino para poder diseñar e implementar planes de restauración efectivos de ecosistemas alterados.

Ciertos grupos de vertebrados que dependen del ambiente acuático para desarrollar su ciclo de vida, como determinadas especies de aves y peces, poseen características apropiadas para ser considerados indicadores biológicos según Noss (1990). En este sentido, se trata de grupos de organismos conspicuos, sensibles a los cambios del ambiente, diversos, con distribuciones amplias en los territorios y relativamente fáciles de identificar y relevar. Por esta razón, han sido utilizados en muchas oportunidades para detectar impactos asociados con el uso de suelo (Karr, 1981; Lantschner y Rusch, 2007; Miserendino et al., 2011).

En la región pampeana existen trabajos que evidencian la pérdida de biodiversidad y calidad ambiental como consecuencia de la urbanización (Bértora et al., 2018; Paracampo et al., 2020; Remes Lenicov et al., 2005), la agricultura (Arias et al., 2020; Codesido y Bilenca, 2011, Solis et al., 2017) y la ganadería (Giorgi et al., 2014; Granitto et al., 2016). No obstante, la información respecto de las alteraciones en los ensambles de aves y peces continúa siendo insuficiente para el establecimiento de líneas de base que permitan el desarrollo de políticas de manejo territorial y rehabilitación ambiental. Por lo tanto, el objetivo de este trabajo se orienta a la evaluación de las comunidades de aves y de peces en arroyos pampeanos con bañados de desborde con distinto tipo de intervención humana, estimando su valor como indicadores biológicos a nivel regional. De 
acuerdo con el enunciado precedente, se espera encontrar diferencias en las estructuras de los ensambles de peces y aves como respuesta a los usos del territorio.

\section{MATERIALES Y MÉTODOS}

\section{Área de estudio}

Los arroyos estudiados pertenecen al grupo de cursos de agua tributarios del estuario del Río de la Plata y están ubicados en la llanura pampeana. Esta región posee un clima templado y húmedo, con temperaturas medias que varían anualmente de 9,9 a $22,4^{\circ} \mathrm{C}$, precipitación anual media de 1,060 mm y evapotranspiración anual media de $783 \mathrm{~mm}$. Dada la escasa pendiente de la región, el flujo de agua es lento con variaciones leves a lo largo del año, excepto luego de lluvias torrenciales cuando ocurren picos de flujo y desbordes (Feijoó et al., 1999; Rodrigues Capítulo et al., 2010). El lecho de estos arroyos es limoso, y sus aguas tienen $\mathrm{pH}$ alcalino, elevada concentración de materia orgánica en suspensión, altas concentraciones de nutrientes y altos valores de oxígeno disuelto (Feijoó et al., 1999). Además, los arroyos de esta zona pueden recibir de manera permanente $o$ semi-permanente aportes de agua subterránea (Deluchi et al., 2005).

Los relevamientos, tanto de aves como de peces, fueron realizados en los sectores superiores de las cuencas de cuatro arroyos con bañados de desborde: Carnaval, del Gato, Cajaravillas y Chubichaminí (Figura 1). Las aves se muestrearon en toda la extensión del bañado, mientras que las muestras de peces se tomaron en puntos cercanos al ingreso y egreso de los arroyos a sus respectivos bañados. El área abarcada por los muestreos de aves y peces en cada arroyo con su bañado se refieren en el documento como sitios.

Los sitios presentan diferencias en cuanto al uso de suelo y grado de intervención humana. Debido a su ubicación periurbana (Per), los sitios correspondientes a los arroyos Carnaval y del Gato (CNV-Per y GAT-Per, respectivamente) son los más afectados por las actividades antrópicas entre los cuatro estudiados. La cuenca del arroyo CNV-Per presenta un uso del territorio

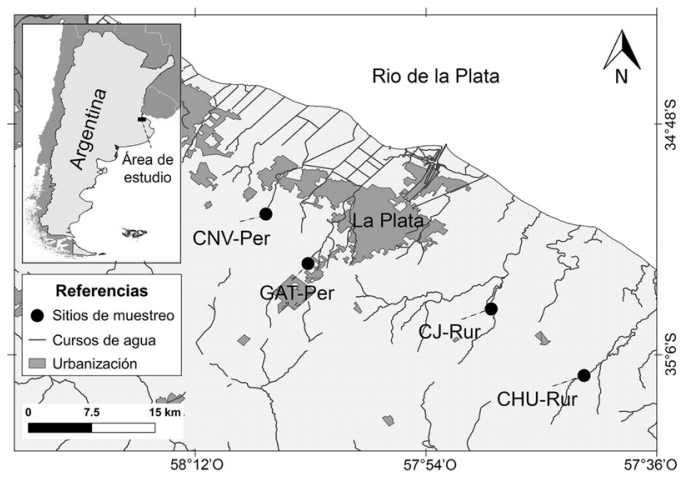

Figura 1. Área de estudio con la ubicación de los sitios de muestreo localizados en sectores periurbanos: Carnaval (CNV-Per) y del Gato (GAT-Per), y sectores rurales: Cajaravilla (CJ-Rur) y Chubichaminí (CHU-Rur).

Figure 1. Study area with peri-urban sampling sites location: Carnaval (CNV-Per) and del Gato (GAT-Per), and rural sampling sites location: Cajaravilla (CJ-Rur) y Chubichaminí (CHU-Rur).

dedicado al cultivo de soja, trigo y maíz en los sectores superiores, actividad que ocupa el $81 \%$ de la cuenca. En contraste, los sectores medios e inferiores exhiben un uso de suelo que varía de periurbano a urbano, representando el $19 \%$ de la superficie de esta cuenca y albergando alrededor de 40000 habitantes. La cuenca del arroyo GAT-Per comprende sectores superiores con agricultura intensiva bajo cubierta y sectores medios e inferiores densamente urbanizados. Este último uso de suelo representa el $38 \%$ de la superficie de la cuenca y concentra el $65 \%$ de los habitantes del partido de La Plata (322000 habitantes). Los bañados de estos arroyos presentan superficies reducidas debido a los cambios hidrológicos relacionados con los dragados y canalizaciones realizados en las últimas décadas. La vegetación en ambos sitios está representada por plantas acuáticas flotantes, sumergidas y arraigadas emergentes, mientras que la vegetación ribereña se encuentra altamente modificada.

Las cuencas de los arroyos Cajaravillas y Chubichaminí se encuentran localizadas en sectores rurales (CJ-Rur y CHU-Rur, respectivamente), con presencia de ganadería extensiva sobre pasturas (menos de 0,7 vacas/ ha) como uso predominante del suelo. Además, en sus cuencas de drenaje se encuentran pequeños centros urbanos que en conjunto 
no superan los 5000 habitantes. La vegetación ribereña de los sitios CJ-Rur y CHU-Rur se caracteriza por el predominio de especies hemicriptófitas, lo cual evidencia una estrecha relación con la actividad ganadera (Zanotti y Gómez, 2019). En cuanto a las características de los bañados, el correspondiente a CJ-Rur tiene la mayor superficie entre los cuatro estudiados. En este sitio los densos rizomas de las macrófitas Typha latifolia (totora), Schoenoplectus californicus (junco) e Iris pseudacorus (lirio), junto al aporte de materia orgánica proveniente de los tallos y hojas senescentes, producen la colmatación de una gran parte del bañado, lo cual restringe los sectores de aguas libres y determina una escasa profundidad de la columna de agua y el predominio del escurrimiento laminar. El sitio CHU-Rur presenta una extensa cobertura de Schoenoplectus californicus, sin embargo, la superficie de aguas libres predomina con respecto al área ocupada por macrófitas, lo cual marca una clara diferencia con el sitio CJ-Rur.

\section{Muestreo y análisis de los datos}

Los sitios se muestrearon en cuatro ocasiones durante el otoño y primavera de los años 2017 y 2018. La estimación de la densidad de las especies de aves se realizó mediante transectas de 1000 m y una hora de duración, en las que se registraron todas las aves asociadas con el ancho del humedal, aquellas que estaban haciendo uso del ambiente y las que volaban siguiendo la dirección del curso de agua (Ralph et al., 1996). Para la nomenclatura se siguió a Remsen et al. (2015), y la identificación se realizó según Ridgely \& Tudor (2009) y Rodríguez Mata et al. (2006). Además, se tipificó a cada especie de ave en relación con su dependencia del ambiente: ave acuática (A.A.) o ave terrestre (A.T.) según el criterio de Martínez (1993).

El muestreo de los ensambles de peces se realizó aplicando el mismo esfuerzo de pesca en todos los sitios de muestreo. La estrategia fue utilizar dos artes de pesca activos que permitieran la exploración y cuantificación de una mayor variedad de ambientes dentro de los arroyos. Las zonas de aguas abiertas se examinaron con una red de arrastre con copo (longitud $15 \mathrm{~m}$; altura 1,1 m; tamaño de malla del ala $10 \mathrm{~mm}$ de distancia entre nudos; copo con longitud $2 \mathrm{~m}$ y tamaño de malla de $5 \mathrm{~mm}$ de distancia entre nudos) en una sección de flujo de $20 \mathrm{~m}$. Los microhábitats compuestos por sectores vegetados se muestrearon con una red manual ("copo") que se arrastró a lo largo de un metro lineal. La red del arte es un tejido con hilos uniformes de monofilamento de polietileno de alta densidad, con una apertura de la malla de $1 \times 1 \mathrm{~mm}$.

La captura de cada sitio y fecha de muestreo fue procesada en el campo donde se registró el número de ejemplares colectados por especie y lance efectuado con cada arte de pesca. Los peces fueron liberados y sólo se conservaron muestras de aquellos que no pudieron ser identificados en el campo. Dichos ejemplares se sacrificaron en una solución de benzocaína en exceso, se fijaron en una solución de formaldehído al 10\% y luego fueron transferidos a etanol al $70 \%$ para su identificación en el laboratorio. Las especies fueron identificadas siguiendo a Almirón et al. (2015), Aquino (1997), Azpelicueta y Braga (1991), Braga, (1993), Casciotta et al. (2005), Reis \& Pereira (2000) y Říčan \& Kullander, (2008). Las actualizaciones en taxonomía se revisaron de Koerber et al. (2017), Mirande \& Koerber (2020) y Terán et al. (2020). Se registró el número de individuos de cada especie para cada muestra discriminando por artes de pesca y se estandarizó mediante la estimación a $\mathrm{m}^{2}(\mathrm{~N})$, considerando el área cubierta por cada arte de pesca como criterio principal. En el caso de las redes manuales, las correcciones y estandarizaciones se aplicaron considerando el porcentaje de la superficie de cada arroyo con aguas libres o vegetadas. Finalmente se estimaron las densidades promedio para cada especie capturada por sitio y fecha de muestreo.

Para ambos grupos taxonómicos, y para cada muestra se registró la riqueza específica (S) y se calculó el índice de diversidad de ShannonWiener $\left(\mathrm{H}^{\prime}\right)$. Se clasificaron los sitios mediante análisis de agrupamientos (CLUSTER) basados en datos de abundancias de aves y peces por separado. Los valores fueron previamente transformados a log10 $(\mathrm{N}+1)$. Se utilizó el índice de similitud de Bray-Curtis para construir las matrices. La significancia de los arreglos obtenidos y las diferencias entre los grupos 
se evaluó mediante ANOSIM. La contribución específica a la similitud de cada grupo y a la disimilitud entre grupos se realizó por SIMPER. Los análisis fueron realizados con el software Primer-e 6.0 (Clarke \& Warwick, 2004).

Finalmente, con el fin de evaluar la respuesta de los ensambles estudiados a los cambios en el uso del suelo, se agruparon las especies en función de su sensibilidad y se examinó la estructura de ambos ensambles mediante el análisis de las abundancias relativas de cada categoría por sitio. Para el agrupamiento, las aves se clasificaron siguiendo a López-Lanús et al. (2008) mientras que la categorización de los peces comprendió cuatro categorías de sensibilidad basadas en estudios desarrollados por diversos autores (Bértora et al., 2018; Bozzetti \& Schulz, 2004; Colautti et al., 2015; Hued \& Bistoni, 2005; Remes Lenicov et al., 2005).

\section{RESULTADOS}

\section{Riqueza y diversidad de los ensambles de aves y peces}

Se registraron un total de 87 especies de aves en todo el programa de muestreo, que corresponden a 31 familias que se distribuyeron en 16 órdenes (Tabla 1, material suplementario). El arroyo CJ-Rur presentó la mayor S con 66 especies, de las cuales un $70 \%$ se correspondió con A.A. El arroyo CHU-Rur presentó 35 especies de aves cuya mayoría (86\%) también correspondieron a especies de A.A. El número total de aves registradas en este estudio para el arroyo GAT-Per fue de 23 (61\% fueron A.A.) y siete especies para el arroyo CNV-Per, de las cuales sólo tres fueron A.A. Las especies Aramus guarauna (carau), Caracara plancus (carancho) e Hymenops perspicillatus (pico de plata) fueron las únicas especies presentes en todos los sitios. Los valores más altos de $\mathrm{H}^{\prime}$ para las aves acuáticas de todos los sitios de muestreo se estimaron en los arroyos rurales, mientras que la máxima estimación para las A.T. se registró en el CJ-Rur. Cabe señalar que para las A.A., $\mathrm{H}^{\prime}$ mostró la menor variabilidad en el sitio $\mathrm{CHU}$ Rur contemplando todo el período de estudio (Figura 2).

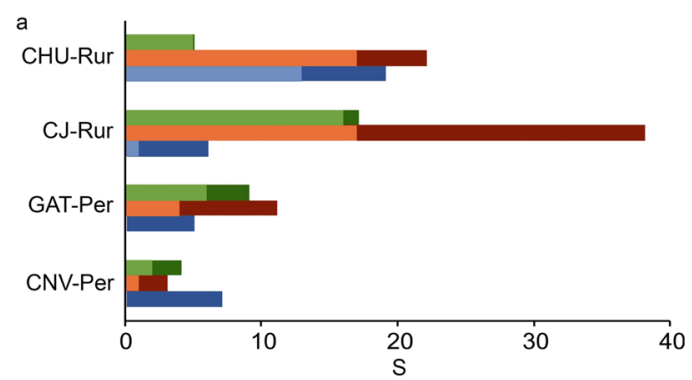

b

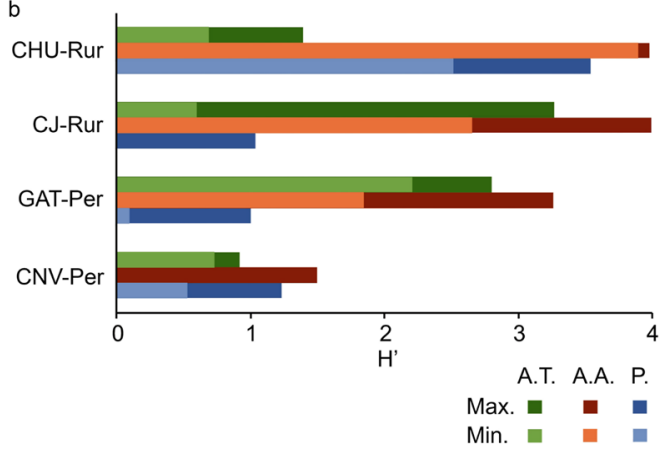

Figura 2. a Valores mínimos (Mín) y máximos (Máx) de riqueza específica (S) y b. del índice de ShannonWiener $\left(H^{\prime}\right)$ de aves terrestres (A.T.), aves acuáticas (A.A.) y peces (P.).

Figure 2. a Minimum and maximum values of specific richness $(S)$ and $\mathbf{b}$. of Shannon-Wiener $\left(H^{\prime}\right)$ diversity index of terrestrial birds (A.T.), aquatic birds (A.A.) and fish assemblages (P.).

En el caso de los peces se capturaron un total de 31 especies, correspondientes a 13 familias y distribuidas en 5 órdenes (Tabla 2, material suplementario). La mayor $\mathrm{S}$ se registró en el sitio CHU-Rur, sumando un total de 19 especies. Los sitios GAT-Per, CNV-Per y CJ-Rur por el contrario, registraron valores de riqueza inferiores a 10 especies $(5,7$ y 6 como máximo, respectivamente). En lo que respecta a $\mathrm{H}^{\prime}$, el sitio CHU-Rur, presentó valores que fueron más del doble de lo observado para los otros sitios (Figura 2).

\section{Estructura de los ensambles de aves y peces}

El análisis de CLUSTER realizado para las A.T. (Figura 3a) separó a los sitios en cuatro grupos (ANOSIM, R global=0,98; $p=0,001$ ). De acuerdo con las comparaciones de pares, 
Disimilitud entre grupos

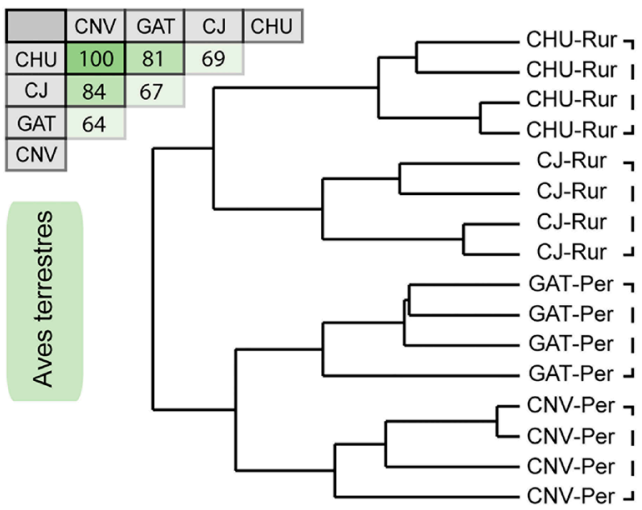

Disimilitud entre grupos

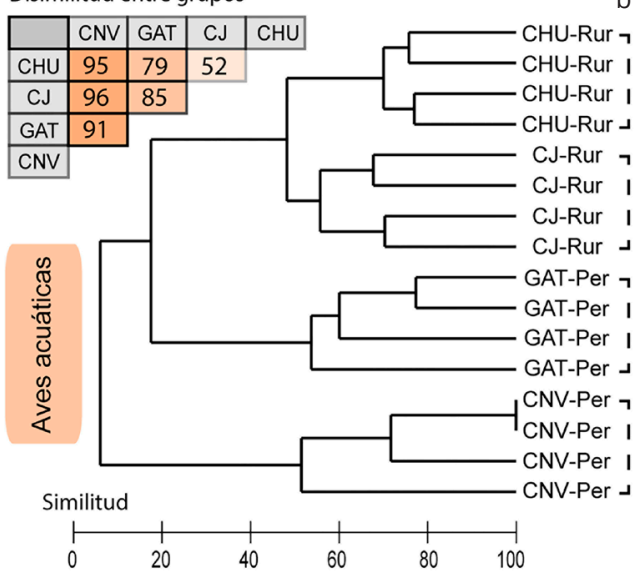

Figura 3. Agrupamientos obtenidos a partir de los análisis de CLUSTER basados en las abundancias de: a. aves terrestres b. las aves asociadas a ambientes acuáticos. Los valores de disimilitud entre grupos obtenidos según SIMPER se consignan a la izquierda de cada CLUSTER.

Figure 3. Groupings obtained from CLUSTER analysis based in abundance of a. terrestrial birds and b. birds related to aquatic environments. Groups dissimilarity values obtained in SIMPER analyses are consigned in each CLUSTER's left.

todos los grupos resultaron estadísticamente diferentes entre sí $(R>0,93 ; p=0,03)$. Según los resultados del análisis SIMPER, el CJ-Rur mostró una elevada contribución de especies de A.T. (11) de las cuales Sturnus vulgaris (estornino pinto), Sicalis luteola (misto), Columba livia (paloma doméstica) y Zonotrichia capensis (chingolo) acumularon el 53\% de la similitud del grupo. Los sitios CNV-Per y CHU-Rur presentaron 2 y 3 especies respectivamente que explicaron la similitud intragrupo, de las cuales
Z. capensis y Troglodytes aedon (ratona común) englobaron la totalidad de la similitud del grupo CNV-Per y S. luteola, Myiopsitta monachus (cotorra) y Nothura maculosa (inambú común) comprendieron la totalidad de la similitud del grupo CHU-Rur. Para el sitio GAT-Per, seis especies contribuyeron a la similitud intragrupo, siendo M. monachus y Furnarius rufus (hornero) las especies determinantes de la similitud del grupo al acumular el $51 \%$ de la misma.

Sobre la base de las abundancias de A.A., el CLUSTER separó a los sitios en cuatro grupos (ANOSIM: $R$ global $=0,95 ; p=0,001$ ) en un arreglo similar al obtenido en el análisis basado en las abundancias de A.T. (Figura 3b). Los grupos resultaron estadísticamente diferentes entre sí de acuerdo con las comparaciones de pares $(R>0,84 ; p=0,03)$. Según los resultados del SIMPER, los sitios con uso de suelo rural presentaron un alto número de especies de aves que contribuyeron de manera relativamente homogénea a la similitud de cada grupo (CJRur, 17 y CHU-Rur, 14). En este sentido, las contribuciones de las especies Pseudoleistes virescens (pecho amarillo), Agelasticus thilius (varillero ala amarilla), Embernagra platensis (verdón), Cranioleuca sulphurifera (curité ocráceo), Pitangus sulphuratus (benteveo común) y Phleocryptes melanops (junquero) acumularon el $47 \%$ de la similitud del grupo CJ-Rur. Para el sitio CHU-Rur el 50\% de la similitud acumulada del grupo lo aportaron Milvago chimango (chimango), A. thilius, Anthus correndera (cachirla común), Geothlypis aequinoctialis (arañero cara negra), $P$. melanops y $P$. sulphuratus. En contraste, las similitudes de los sitios de áreas periurbanas estuvieron explicadas por un menor número de especies (CNV-Per, 3 y GATPer, 6). Así, A. guarauna contribuyó con el 64\% a la similitud del grupo CNV-Per, mientras que Vanellus chilensis (tero), P. sulphuratus y Anas flavirostris (pato barcino) acumularon el $73 \%$ de la similitud del grupo GAT-P.

El análisis de CLUSTER basado en las densidades de peces (Figura 4) separó al sitio CHU-Rur de CNV-Per, GAT-Per y CJ-Rur, los cuales integraron un mismo grupo (ANOSIM, R global=0,99, $p=0,005)$. El resultado del SIMPER evidenció que la contribución de las especies Cheirodon interruptus (mojarra colita negra), Pseudocorynopoma doriae (mojarra de velo), 
Cnesterodon decemmaculatus (panzudito), Diapoma terofali (mojarrita) y Hyphessobrycon meridionalis (mojarra cola roja) acumularon el $85 \%$ de la similitud del grupo para CHU-Rur, a diferencia del grupo conformado por el resto de los sitios, donde solo el panzudito acumuló el $87 \%$ de la similitud del grupo.

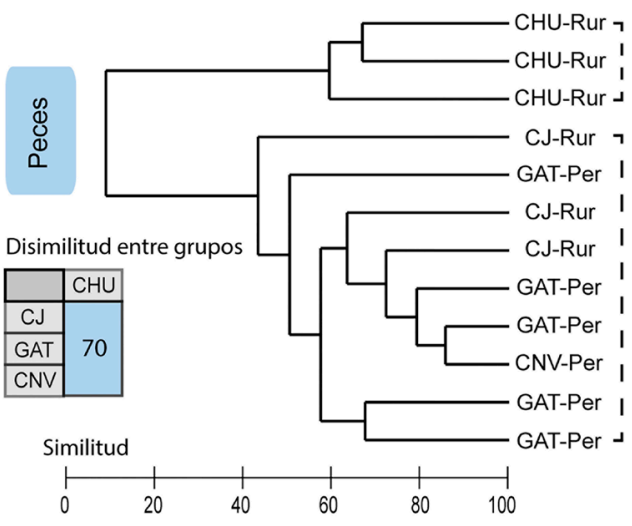

Figura 4. Agrupamientos obtenidos a partir de los CLUSTER basados en las abundancias de los peces. Los valores de disimilitud entre grupos obtenidos según SIMPER se consignan a la izquierda del CLUSTER.

Figure 4. Groupings obtained from CLUSTER analysis based on fish abundance. Groups dissimilarity values obtained in SIMPER analysis are consigned in the CLUSTER's left.

Al analizar la constitución de los ensambles de aves en función de su sensibilidad a la intervención humana del ambiente (Figura 5a), se observaron respuestas contrastantes entre

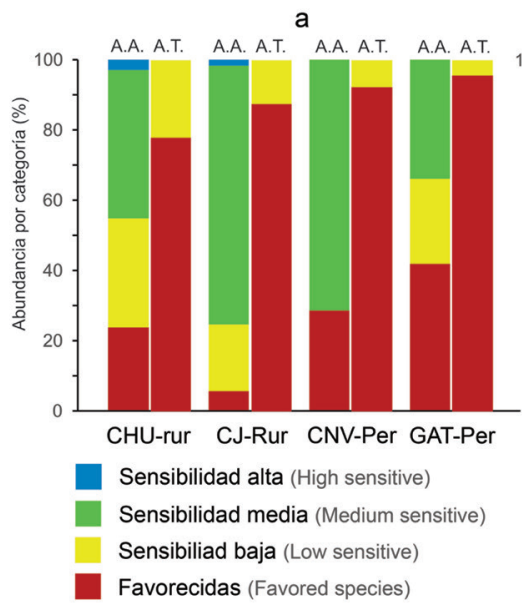

los sitios. En el caso de las aves acuáticas existió un predominio de especies con diversos grados de sensibilidad en los sitios con uso de suelo rural, pero los porcentajes de las que se favorecen con las perturbaciones de origen antrópico resultaron bajos. Por el contrario, en los sitios localizados en sectores periurbanos las especies de sensibilidad alta no estuvieron presentes y los mayores porcentajes de abundancia relativa correspondieron a las especies que se favorecen con las modificaciones antrópicas y las de menor sensibilidad. En el caso de las A.T., se observó una clara dominancia de aquellas que se favorecen con las perturbaciones antrópicas indistintamente entre los sitios. El análisis de las abundancias relativas de los peces en función de sus tolerancias al impacto antrópico (Figura 5b), mostraron un claro patrón de diferenciación entre el CHU-Rur respecto del resto. Dicha diferencia se basó fundamentalmente en la presencia en más de un $50 \%$ de especies sensibles o poco tolerantes en la constitución del ensamble de CHU-Rur y en la clara dominancia de especies muy tolerantes en el resto de los sitios.

\section{DISCUSIÓN}

Los resultados obtenidos en este estudio mostraron que existen importantes diferencias entre los ensambles, tanto de aves como de peces, de los arroyos y sus bañados de desborde,

b

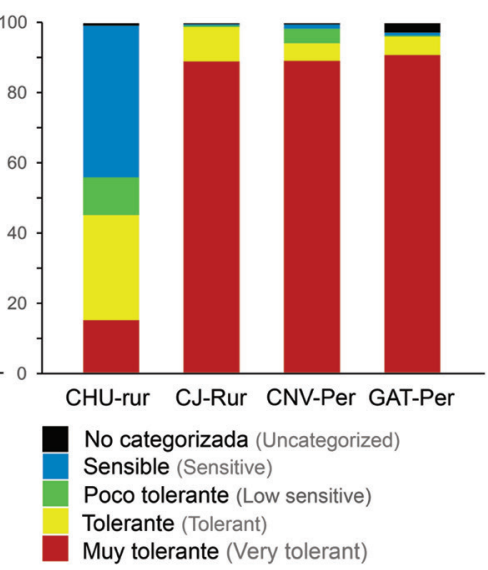

Figura 5. Abundancia relativa en función de su tolerancia a los disturbios antrópicos para los sitios estudiados. a. aves acuáticas (A.A.) y aves terrestres (A.T.) b. peces.

Figure 5. Relative abundance in function of their tolerance to anthropic disturbances for the study sites. a. Aquatic birds (A.A.) and terrestrial birds (A.T.) b. fishes. 
según la cuenca de pertenencia. Weller (1999) y Batzer \& Sharitz (2014) postulan que estos grupos de vertebrados se encuentran entre los más importantes indicadores de la salud y funcionamiento de los ecosistemas acuáticos. En este estudio se observó que en aquellos arroyos y bañados asociados donde se registraron los valores más bajos de riqueza y de diversidad, la intervención humana es más intensa, y tanto los cauces como el uso del territorio han sufrido las mayores modificaciones. No obstante, en cada subgrupo conformado por los sitios con uso de suelo rural por un lado y periurbano por otro, se observaron diferencias al comparar sus valores de riqueza específica y de diversidad.

Si bien los arroyos de zonas rurales presentan un menor grado de perturbación, el número de especies de aves registradas fue más alto en CJ-Rur que CJ-Rur. Teniendo en cuenta que en ambos ambientes rurales la riqueza de aves acuáticas fue similar, la diferencia entre ambos sitios estuvo marcada por la mayor riqueza de aves terrestres en CJ-Rur. En este sentido, la diferencia entre la avifauna terrestre de ambos sitios puede tomarse como evidencia de una mayor diversidad de hábitats para este tipo de aves en el sitio CJ-Rur. La presencia de arboledas conspicuas alrededor del bañado posibilita la disponibilidad de estratos arbóreos en el sitio CJ-Rur, y la gran extensión de su bañado genera la existencia de una amplia franja de transición entre los ambientes acuáticos y terrestres, aumentando la complejidad estructural de este sistema. Este fenómeno resulta consistente con otros estudios donde se ha relacionado el aumento de diversidad y riqueza de aves con el aumento de la superficie de bañado y áreas forestadas en la cuenca (Mensing et al., 1998). Sin embargo, el ensamble de peces tuvo una respuesta diferente al de aves, ya que tanto la riqueza como la diversidad resultaron superiores en CHU-Rur respecto de CJ-Rur. Por lo expuesto en la descripción de ambos arroyos, la diferencia tendría que ver fundamentalmente con las características de los cursos de agua. En este sentido, si bien tanto CHU-Rur como CJ-Rur poseen extensos bañados de desborde, el área correspondiente a aguas libres es comparativamente mayor al ocupado por vegetación y materia orgánica acumulada en el sitio CHU-Rur. En contraposición, en CJ-Rur la proporción de bañado ocupado por vegetación y materia orgánica es considerablemente mayor, reduciendo al sector de aguas libres a una estrecha franja de escasa profundidad en el centro del cauce. Dadas estas características, el sitio CJ-Rur es apto solo para albergar algunas especies de peces que presentan adaptaciones para vivir en este tipo de ambiente, como por ejemplo $C$. decemmaculatus que puede captar el oxígeno disuelto de la capa superior del agua (Kramer \& Mehegan, 1981). El mencionado fenómeno es consistente con otros estudios realizados en humedales, donde se reportó el aumento de la abundancia de peces en función a la proporción de superficie representada por aguas libres (Mensing et al., 1998).

Los ensambles de aves y peces de los sitios periurbanos correspondieron a situaciones de mayor modificación antrópica con menor riqueza y diversidad, aunque también se distinguieron entre sí. Los valores más bajos para ambos grupos de aves se registraron en CNV-Per, lo que podría vincularse con una reducción en la diversidad de hábitats como consecuencia del uso de suelo agrícola extensivo. A pesar de ello, los menores valores de riqueza y diversidad de ensambles de peces fueron registrados en GAT-Per, donde el uso de suelo predominante fue agrícola intensivo. Esta disparidad, podría sugerir la existencia de un impacto diferencial por parte de ambos sistemas de agricultura sobre los ensambles de peces $y$ aves $y$, en consecuencia, sobre los sistemas acuático y terrestre lindero al arroyo, hecho que merece ser explorado con mayor profundidad en futuros estudios.

Los resultados del presente estudio posibilitan discriminar cuales son las especies que caracterizan a cada uno de los sitios. En el caso de las aves terrestres, las especies responsables de la similitud intragrupo fueron propias de cada uno de ellos. A diferencia del resto de los arroyos, la similitud en CJ-Rur estuvo dada por varias especies, evidenciando que la mayor superficie del bañado favorece una zona de transición más extendida entre los ambientes terrestres y acuáticos, que ejercería una fuerte influencia sobre la estructura de este ensamble. En lo que respecta a las aves 
acuáticas, los sitios ubicados en áreas rurales presentaron identidad propia y ensambles cuyas similitudes intragrupo se explicaron por composiciones en las que estuvieron involucradas varias especies. Probablemente la menor intervención humana en la cuenca y el desarrollo de dos ambientes acuáticos con diferencias fisonómicas e hidrológicas influyan fuertemente en sus estructuras. En cuanto a los sitios de sectores periurbanos, los resultados confirmaron el empobrecimiento de estos ensambles como consecuencia de la intervención antrópica.

En el análisis de composición relativa de los ensambles en función de las abundancias de las especies con diferente sensibilidad al deterioro ambiental, en general, se observó que tanto en CHU-Rur como en CJ-Rur las aves acuáticas tuvieron buena representación de especies con baja tolerancia, mientras que CNV-Per y GAT-Per estuvieron dominados por las menos sensibles y con una menor cantidad de especies. Esto también se observó en el caso de los peces, donde CJ-Rur formó parte del grupo de los sitios habitados por especies categorizadas como muy tolerantes, debido a las características del sitio ya discutidas. Este fenómeno de reducción de especies y predominio de aquellas tolerantes en función del incremento de la presión antrópica sobre el ambiente ha sido documentado en otros sitios del planeta y fue denominado homogeneización biótica (Fuller et al., 2015). Un caso particular en este análisis lo representaron las aves terrestres cuyos ensambles estuvieron indistintamente dominados por especies que se favorecen con la intervención humana sobre el ambiente.

En este trabajo se ha mostrado que las modificaciones producidas por las actividades humanas en el territorio y particularmente en las cuencas hidrográficas, generan cambios en los ensambles de especies que dependen del ambiente acuático para desarrollar su ciclo de vida. Esto podría tener consecuencias directas sobre el funcionamiento de estos ecosistemas. En este sentido, se ha comprobado que estas intervenciones, en muchos casos, han acelerado la degradación del hábitat en los bañados de desborde de los cursos de agua (Li et al., 2019; Richter et al., 2010; Opperman et al., 2009).
Según diversos autores (Albert et al., 2020; Fischer \& Lindenmayer, 2007), esta situación está causando la pérdida de la biodiversidad en ambientes dulceacuícolas, lo cual resulta crítico ya que sostienen una alta riqueza de especies en relación con su limitada extensión.

El estudio en simultáneo de cuatro arroyos con bañados sometidos a diferente grado de intervención humana nos permitió registrar de qué manera en una misma región los cambios inducidos por el hombre condicionan la estructura de los ensambles de vertebrados conspicuos como las aves y los peces, posiblemente por la reducción selectiva en la capacidad de colonización, establecimiento y/o permanencia de las poblaciones que los configuran. Lo observado coincide con otros estudios en los que se comprobó que debido a la pérdida de disponibilidad de hábitat (Pardini et al., 2010) y al incremento del aislamiento de los parches que mantienen condiciones para el desarrollo de las poblaciones (Amoros \& Bornette, 2002), tanto la riqueza, como la diversidad y la abundancia de organismos se reduce y es reemplazada por ensambles compuestos principalmente por especies más tolerantes a los disturbios y con capacidades de dispersión alta (Edge et al., 2017, van Puijenbroek et al., 2018). Este fenómeno se ha vinculado con la desaparición local de las poblaciones por reducción de su tamaño (paradigma de las poblaciones pequeñas, Sæther et al., 1996) y/o la pérdida de su viabilidad debido a la imposibilidad de recibir inmigrantes a causa del aislamiento (paradigma de las metapoblaciones, Hanski, 1999). Más allá de que nuestro diagnóstico acerca de la situación de los ensambles de aves y peces de los arroyos estudiados podría explicarse en buena medida sobre la base de las teorías planteadas, lo cierto es que en los sitios de mayor impacto humano han experimentado un profundo deterioro en su estructura.

El trabajo realizado representa un valioso aporte, no sólo por su carácter diagnóstico, sino también porque provee un enfoque multi-taxa que facilita la evaluación de la calidad ambiental sobre la base de la composición y estructura de sus ensambles de aves y peces, brindando herramientas novedosas para monitorear 
las eventuales medidas de remediación que se implementen en ecosistemas acuáticos pampeanos.

\section{AGRADECIMIENTOS}

Los/as autores/agradecen a los/as revisores por su tiempo y esfuerzo en generar aportes que han mejorado sustancialmente el manuscrito. El presente estudio fue realizado mediante el financiamiento de la Agencia Nacional de Promoción Científica y Tecnológica, Argentina; PICT 2015-1342 y el Consejo Nacional de Investigaciones Científicas y Técnicas (CONICET), Argentina; PI-UE 22920160100049CO. Este trabajo representa la contribución científica número 1182 del Instituto de Limnología "Dr. Raúl A. Ringuelet".

\section{REFERENCIAS}

Abell, R., Thieme, M. L., Revenga, C., Bryer, M., Kottelat, M., Bogutskaya, N., Coad, B., Mandrak, N., Balderas, S. C., Bussing, W., Stiassny, M. L. J., Skelton, P., Allen, G. R., Unmack, P., Naseka, A., Ng, R., Sindorf, N., Robertson, J., Armijo, E., Higgins, J. V., Heibel, T. J., Wikramanayake, E., Olson, D., López, H .L., Reis, R. E., Lundberg, J. G., Sabaj Pérez, M. H. \& Petry, P. (2008). Freshwater Ecoregions of the World: A New Map of Biogeographic Units for Freshwater Biodiversity Conservation. BioScience, 58, 403-414.

Albert, J. S., Destouni, G., Duke-Sylvester, S. M., Magurran, A. E., Oberdorff, T., Reis, R. E., Winemiller, K. R. \& Ripple, W. J. (2020). Scientists' warning to humanity on the freshwater biodiversity crisis. Ambio.

Allan, J. D. (2004). Landscapes and Riverscapes: The Influence of Land Use on Stream Ecosystems. Annual Review of Ecology, Evolution, and Systematics, 3 257-284.

Almirón, A., Casciotta, J., Piálek, L., Doubnerová, K. \& Říčan, O. (2015). Oligosarcus amome
(Ostariophysi: Characidae), a new species from the Río Uruguay basin, Misiones, Argentina. Zootaxa, 391(1), 581-590.

Amoros, C. \& Bornette, G. (2002). Connectivity and biocomplexity in waterbodies of riverine floodplains. Freshwater Biology, 47, 761-776.

Aquino, A. E. (1997). Las especies de Hypoptomatinae (Pisces, Siluriformes, Loricariidae) en la Argentina. Revista de Ictiología, 5(1-2), 5-21.

Arias, M., Scalise, A., Solis, M., Paracampo, A., Indaco, M., Fanelli, S., Mugni, H. \& Bonetto, C. (2020). Horticulture affects macroinvertebrate assemblages in adjacent streams (Buenos Aires, Argentina). Knowledge \& Management of Aquatic Ecosystems, 421(1), 5.

Azpelicueta, M. M. y Braga, L. (1991). Los Curimátidos en Argentina. En: Castellanos Z.A. (Ed.). Fauna de agua dulce de la República Argentina (pp. 1-55). Argentina: Profadu CONICET.

Bateman, I. J., Harwood, A. R., Mace, G. M., Watson, R. T., Abson, D. J., Andrews, B., Binner, A., Crowe, A., Day, B. H., Dugdale, S., Fezzi, C., Foden, J., Hadley, D, Haines-Young, R., Hulme, M., Kontoleon, A., Lovett, A. A., Munday, P., Pascual, U., Paterson, J., Perino, G., Sen, A., Siriwardena, G., Van Soest, D. \& Termansen, M. (2013). Bringing ecosystem services into economic decision-making: Land use in the United Kingdom. Science, 05, 45-50.

Batzer, D. \& Sharitz, R. (Eds.). (2014). Ecology of Freshwater and Estuarine Wetlands. University of California Press.

Bértora, A., Grosman, F., Sanzano, P. y Rosso, J. J. (2018). Composición y estructura de los ensambles de peces en un arroyo pampeano con uso de suelo contrastante. Revista del Museo Argentino de Ciencias Naturales nueva serie, 20(1), 11-22.

Blann, K. L., Anderson, J. L., Sands, G. R. \& Vondracek, B. (2009). Effects of agricultural drainage on aquatic ecosystems: A review. Critical Reviews in Environmental Science and Technology, 39, 909-1001. 
Bozzetti, M. \& Schulz, U. H. (2004). An index of biotic integrity based on fish assemblages for subtropical streams in southern Brazil. Hydrobiologia, 529(1-3), 133-144.

Braga, L. (1993). Los Anostomidae (Pisces, Characiformes) de Argentina. En: Castellanos Z. A. (Ed.). Fauna de agua dulce de la República Argentina (pp. 5-45). Argentina: Profadu, CONICET.

Burcher, C. L. \& Benfield, E. F. (2006). Physical and biological responses of streams to suburbanization of historically agricultural watersheds. Journal of the North American Benthological Society, 25(2), 356-369.

Casciotta, J., Almirón, A. y Bechara, J. (2005). Peces del Iberá - hábitaty diversidad. La Plata: Grafikar. Fundación Ecos, UNLP y UNNE.

Chace, J. F. \& Walsh, J. J. (2006). Urban effects on native avifauna: a review. Landscape and Urban Planning, 74(1), 46-69.

Clarke, K. R. \& Warwick, R. M. (2001). Change in marine communities. An approach to statistical analysis and interpretation. United Kingdom: Plymouth Marine Laboratory.

Codesido, M. y Bilenca, D. N. (2011). Los pastizales y el servicio de soporte de la biodiversidad: Respuesta de la riqueza de aves terrestres a los usos de la tierra en la provincia de Buenos Aires. En: Laterra, P., Jobbágy, E. G., y Paruelo, J. M (Eds). Valoración de servicios ecosistémicos: Conceptos, Herramientas y aplicaciones para el ordenamiento territorial (pp. 511-526). Buenos Aires: INTA.

Colautti, D., Brancolini, F., García, I., Garcia de Souza, J., Jensen, R., Maiztegui, T., Maroñas, M., Paracampo, A., Paredes del Puerto, J. M., Protogino, L. y Sendra, E. (2015). Monitoreo de la ictiofauna en cursos de agua superficial de la cuenca hidrográfica Matanza-Riachuelo. ACUMAR - CEC Nro. 4 - PECES.

Daily, G. C., Polasky, S., Goldstein, J., Kareiva, P. M., Mooney, H. A., Pejchar, L., Ricketts, T. H., Pejchar, L., Salzman, J. \& Shallenberger, R. (2009). Ecosystem services in decision making: time to deliver. Frontiers in Ecology and the Environment, 7(1), 21-28.

De Groot, R. S., Alkemade, R., Braat, L., Hein, L. \& Willemen, L. (2010). Challenges in integrating the concept of ecosystem services and values in landscape planning, management and decision making. Ecological complexity, 7(3), 260-272.

Deluchi, M., Kruse, E. E., Laurencena, P. C., Rojo, A. y Carol, E. S. (2005). Variaciones de los niveles freáticos y su relación con el agua superficial en La Plata (Provincia de Buenos Aires). En: IV Congreso Hidrogeológico Argentino y II Seminario Hispano-Latinoamericano sobre temas actuales de Hidrología Subterránea. Río Cuarto, Córdoba, 25 al 28 de octubre de 2005. Recuperado en: http://sedici.unlp.edu.ar/handle/10915/26567 (fecha de acceso: 02/09/2020)

Dudgeon, D., Arthington, A. H., Gessner, M. O., Kawabata, Z. I., Knowler, D. J., Lévêque, C., Naiman, R. J., Prieur-Richard, A. H., Soto, D., Stiassny, M. L. J. \& Sullivan, C. A., (2006). Freshwater biodiversity: importance, threats, status and conservation challenges. Biological Reviews, 81, 163-182.

Edge, C. B., Fortin, M. J., Jackson, D. A. Lawrie, D., Stanfield, L. \& Shrestha, N. (2017). Habitat alteration and habitat fragmentation differentially affect beta diversity of stream fish communities. Landscape Ecology, 32, 647-662.

Feijoó, C. S., Giorgi, A., García, M. E. \& Momo, F. (1999). Temporal and spatial variability in streams of a pampean basin. Hydrobiologia, 394, 41-52.

Fischer, J. \& Lindenmayer, D. B. (2007). Landscape modification and habitat fragmentation: a synthesis. Global Ecology and Biogeography, 16(3), 265-280.

Fuller, M. R., Doyle, M. W. \& Strayer, D. L. (2015). Causes and consequences of habitat fragmentation in river networks. Annals of the New York Academy of Sciences, 1355(1): 31-51.

Gafny, S., Goren, M. \& Gasith, A. (2000). Habitat condition and fish assemblage structure in a coastal mediterranean stream (Yarqon, Israel) 
receiving domestic effluent. Hydrobiologia, 422/423, 319-330.

Giorgi, A., Rosso, J. J. y Zunino, E. (2014). Efectos de la exclusión de ganado sobre la calidad ambiental de un arroyo pampeano. Biología Acuática, 30, 133-140.

Goldstein, B. D., Kriesky, J. \& Pavliakova, B. (2012). Missing from the table: role of the environmental public health community in governmental advisory commissions related to Marcellus Shale drilling. Environmental Health Perspectives, 120(4), 483-486.

Granitto, M., Rosso, J. J., Boveri, M. B. y Rennella, A. M. (2016). Impacto del uso del suelo sobre la condición de ribera en arroyos pampeanos y su relación con la estructura de la comunidad de peces. Biologia Acuatica, 31, 19-27.

Hanski, I. (1999). Habitat connectivity, habitat continuity, and metapopulations in dynamic landscapes. Oikos, 87, 209-219.

Helms, B. S., Feminella, J. W. \& Pan, S. (2005). Detection of biotic responses to urbanization using fish assemblages from small streams of western Georgia, USA. Urban Ecosystems, 8, 39-57.

Hued, A. C. \& Bistoni, M. D. L. Á. (2005). Development and validation of a Biotic Index for evaluation of environmental quality in the central region of Argentina. Hydrobiologia, 543(1), 279-298.

Karr, J.R. (1981). Assessment of biotic integrity using fish communities. Fisheries, 6, 21-27.

Koerber, S., Litz, T. O. \& Mirande, J. M. (2017). Supplement to Checklist of the freshwater fishes of Argentina. Ichthyological Contributions of Peces Criollos, 55, 1-1.

Kramer, D. L. \& Mehegan, J. P. (1981). Aquatic surface respiration, an adaptive response to hypoxia in the guppy, Poecilia reticulata (Pisces, Poeciliidae). Environmental Biology of Fishes, 6, 299-313.

Lantschner, M. V. y Rusch, V. (2007). Impacto de diferentes disturbios antrópicos sobre las comunidades de aves de bosques y matorrales de Nothofagus antarctica en el NO Patagónico. Ecología Austral, 17(1), 99-112.
Li, B., Tan, W., Wen, L., Zhao, X., Peng, B., Yang, J., Lu, C., Wang, Y. \& Lei, G. (2019). Anthropogenic habitat alternation significantly decreases a- and b-diversity of benthopelagic metacommunity in a large floodplain lake. Hydrobiologia, 847, 293-307.

López-Lanús, B., Grilli, P., Coconier, E., Di Giacomo, A. y Banchs, R. (2008). Categorización de las aves de la Argentina según su estado de conservación. Informe de Aves Argentinas /AOP y Secretaría de Ambiente y Desarrollo Sustentable. Buenos Aires: Aves Argentinas AOP.

Maes, J., Teller, A., Erhard, M., Liquete, C., Braat, L., Berry, P., Egoh, B., Puydarrieux, P., Fiorina, C., Santos, F., Paracchini, M. L., Keune, H., Wittmer, H., Hauck, J., Fiala, I., Verburg, P. H., Condé, S., Schägner, J. P., San Miguel, J., Estreguil, C., Ostermann, O. Barredo, J. I., Pereira, H. M., Stott, A., Laporte, V., Meiner, A., Olah, B., Royo Gelabert, E., Spyropoulou, R., Petersen, J. E., Maguire, C., Zal, N., Achilleos, E., Rubin, A., Ledoux. L., Brown, C., Raes, C., Jacobs, S., Vandewalle, M., Connor, D. \& Bidoglio, G. (2013). Mapping and assessment of ecosystems and their services. An analytical framework for ecosystem assessments under action 5 of the EU biodiversity strategy to 2020. Luxembourg: Publications office of the European Union.

Martínez, M. M. (1993). Las Aves y la Limnología. En: Boltovskoy, A. y López, H. L. (Eds). Conferencias de Limnología. Instituto de Limnología "Dr. R.A. Ringuelet" (pp. 127-142). La Plata: Instituto de Limnología “Dr. R.A. Ringuelet”.

Mc Garrigle, M. L. (2014). Assessment of small water bodies in Ireland. Biology and Environment Proceedings of the Royal Irish Academy, 114B, 119-128.

Mensing, D. M. Galatowitsch, S. M. \& Tester, J. R. (1998). Anthropogenic effects on the biodiversity of riparian wetlands of a northern temperate landscape. Journal of Environmental Management, 53(4), 349-377.

Mirande, J. M. \& Koerber, S. (2020). Checklist of the Freshwater Fishes of Argentina. 2nd edition. (CLOFFAR-2). Ichthyological Contributions of Peces Criollos, 72, 1-81. 
Miserendino, M. L., Casaux, R., Archangelsky, M., Di Prinzio, C. Y., Brand, C. \& Kutschker, A. M. (2011). Assessing land-use effects on water quality, in-stream habitat, riparian ecosystems and biodiversity in Patagonian northwest streams. Science of the Total Environment, 409(3), 612-624.

Noss, R. F. (1990). Indicators for monitoring biodiversity: A hierarchical approach. Conservation Biology, 4, 355-364.

Opperman, J. J., Galloway, G. E., Fargione, J., Mount, J. F., Richter, B. D. \& Secchi, S. (2009). Sustainable floodplains through large-scale reconnection to rivers. Science, 326,1487-1488.

Paracampo, A., Marrochi, N., García, I., Maiztegui, T., Carriquiriborde, P., Bonetto, C. \& Mugni, H. (2020). Fish assemblages of Pampean streams (Buenos Aires, Argentina): relationships with abiotic and anthropogenic variables. Anais da Academia Brasileira de Ciências, 92(2) e20190476.

Pardini, R., de Arruda Bueno, A., Gardner, T. A., Prado, P. I. \& Metzger, J. P. (2010). Beyond the fragmentation threshold hypothesis: Regime shifts in biodiversity across fragmented landscapes. PLOS ONE, 5(10): e13666.

Ralph, C. J., Geupel, G. R., Pyle, P., Martin, T. E., DeSante, D. F. y Milá, B. (1996). Manual de métodos de campo para el monitoreo de aves terrestres. Washington, DC: US Department of Agriculture.

Reis, R. E. \& Pereira, E. H. (2000). Three new species of the loricariid catfish genus Loricariichthys (Teleostei: Siluriformes) from southern South America. Copeia, 2000 (4), 1029-1047.

Remes Lenicov, M., Colautti, D. C. y López, H. L. (2005). Ictiofauna de un ambiente lótico suburbano: el arroyo Rodríguez (Buenos Aires, Argentina). Biología Acuática, 22, 223-230.

Remsen, J. V. Jr, Areta, J. I., Cadena, C. D., Jaramillo, A., Nores, M., Pacheco, J. F., PrezEmn, J., Robbins, M. B., Stiles, F. G., Stotz, D. F. \& Zimmer, K. J. (2015). A classification of the bird species of South America. American Ornithologists Union.
Říčan, O. \& Kullander, S. O. (2008). The Australoheros (Teleostei: Cichlidae) species of the Uruguay and Paraná River drainages. Zootaxa, 1724(1), 1-51.

Richards, C., Johnson, L.B. \& Host, G.E. (1996). Landscape-scale influences on stream habitats and biota. Canadian Journal Fisheries and Aquatic Science, 53, 295-311.

Richter, B. D., Postel, S., Revenga, C., Scudder, T., Lehner, B., Churchill, A. \& Chow, M. (2010). Lost in development's shadow: The downstream human consequences of dams. Water Alternatives, 3, 14-42.

Ridgely, R. S. \& Tudor, G. (2009). Field guide to the songbirds of South America. The Passerines. Austin: University of Texas Press.

Rodrigues Capítulo, A., Gómez, N., Giorgi, A. \& Feijoó, C. (2010). Global changes in pampean lowland streams (Argentina): Implications for biodiversity and functioning. Hydrobiologia, 657(1), 53-70.

Rodríguez Mata, J., Erize, F. y Rumboll, M. (2006) Guía de campo Collins. Aves de Sudamérica, no Passeriformes. Buenos Aires: Harper Collins Publishers.

Roth, N. E., Allan, J. D. \& Erickson, D. L. (1996). Landscape influences on stream biotic integrity assessed at multiple spatial scales. Landscape Ecology, 11, 141-156.

Sæther, B. E., Ringsby, T. H. \& Røskaft, E. (1996). Life history variation, population processes and priorities in species conservation: towards a reunion of research paradigms. Oikos, 77, 217-226.

Solis, M., Mugni, H., Fanelli, S. \& Bonetto, C. (2017). Effect of agrochemicals on macroinvertebrate assemblages in Pampasic streams, Buenos Aires, Argentina. Environmental Earth Sciences, 76(4), 180.

Steedman, R. J. (1988). Modification and Assessment of an Index of Biotic Integrity to Quantify Stream Quality in Southern Ontario. Canadian Journal Fisheries and Aquatic Science, 45, 492-501. 
Strayer, D. L. \& Dudgeon, D. (2010). Freshwater biodiversity conservation: Recent progress and future challenges. Journal of the North American Benthological Society, 29(1), 344-358.

Terán, G. E., Benitez, M. F. \& Mirande, J. M. (2020). Opening the Trojan horse: phylogeny of Astyanax, two new genera and resurrection of Psalidodon (Teleostei: Characidae). Zoological Journal of the Linnean Society, XX, 1-18.

van Puijenbroek, P. J., Buijse, A. D., Kraak, M. H. \& Verdonschot, P. F. (2018). Species and river specific effects of river fragmentation on European anadromous fish species. River Research and Applications, 35, 68-77.
Weller, M. W. (1999). Wetland birds: habitat resources and conservation implications. Cambridge: University Press.

Zanotti, C. y Gómez, N. (2019). Caracterización de bañados de desborde fluvial a partir de inventarios florísticos. En: Cortelezzi, A., Entraigas, I., Grosman, F. y Masson, I. (Eds). Encuentro de saberes para la gestión responsable de ecosistemas acuáticos pampeanos (pp. 116 118). Azul: Universidad Nacional del Centro de la Provincia de Buenos Aires. 